\title{
Monocyte, Activated Killer
}

National Cancer Institute

\section{Source}

National Cancer Institute. Monocyte, Activated Killer. NCI Thesaurus. Code C12548.

A monocyte that has been activated for cytotoxicity by gamma interferon. 